\title{
Erratum to: First- and second-order optimality conditions for multiobjective fractional programming
}

\author{
P. Q. $\operatorname{Khanh}^{1}$ - L. T. Tung ${ }^{2}$
}

Published online: 25 March 2015

(C) Sociedad de Estadística e Investigación Operativa 2015

\section{Erratum to: TOP \\ DOI 10.1007/s11750-014-0347-7}

A mistake has slipped in the Acknowledgments. The correct version is given below.

Acknowledgments This work was supported by National Foundation for Science and Technology Development (NAFOSTED) under grant number 101.01-2014.62. A part of it was completed when the authors stayed as research visitors at Vietnam Institute for Advanced Study in Mathematics (VIASM), whose hospitality is gratefully acknowledged. The second author is supported partially also by Cantho University. The authors are much indebted to the anonymous referees for their valuable remarks and suggestions.

The online version of the original article can be found under doi:10.1007/s11750-014-0347-7.

L. T. Tung

lttung@ctu.edu.vn

P. Q. Khanh

pqkhanhus@gmail.com

1 Department of Mathematics, International University, Vietnam National University Hochiminh City, Linh Trung, Thu Duc, Hochiminh City, Vietnam

2 Department of Mathematics, College of Natural Sciences, Cantho University, Cantho, Vietnam 